\title{
Managing COPD with expiratory or inspiratory pressure load training based on a prolonged expiration pattern
}

Keisuke Miki, Kazuyuki Tsujino, Mari Miki, Kenji Yoshimura, Hiroyuki Kagawa, Yohei Oshitani, Kiyoharu Fukushima, Takanori Matsuki, Yuji Yamamoto and Hiroshi Kida

Affiliation: Dept of Respiratory Medicine, National Hospital Organization Osaka Toneyama Medical Center, Toyonaka, Japan.

Correspondence: Keisuke Miki, Dept of Respiratory Medicine, National Hospital Organization Osaka Toneyama Medical Center, 5-1-1 Toneyama, Toyonaka, Osaka, 560-8552, Japan. E-mail: miki.keisuke.pulamail.hosp.go.jp

\section{ABSTRACT}

Background: Exertional prolonged expiration should be identified as a therapeutic target in COPD. The efficacy of expiratory or inspiratory pressure load training (EPT/IPT) based on the degree of prolonged expiration was investigated.

Methods: A total of 21 patients with COPD were divided into two groups according to the exertional change in the inspiratory duty cycle $\left(T_{\mathrm{I}} / T\right.$ tot). For 12 weeks, patients whose exertional $T_{\mathrm{I}} /$ tot decreased received EPT (EPT group, $\mathrm{n}=11$, mean percentage forced expiratory volume in $\left.1 \mathrm{~s}\left(\% \mathrm{FEV}_{1}\right), 32.8 \%\right)$ and those whose exertional $T_{\mathrm{I}} /$ tot increased received IPT (IPT group, $\mathrm{n}=10$, mean $\% \mathrm{FEV}_{1}, 45.1 \%$ ).

Results: The therapeutic responses were as follows. In both groups, endurance time $(\mathrm{EPT},+5.7 \mathrm{~min}$, $\mathrm{p}<0.0001$; IPT, $+6.1 \mathrm{~min}, \mathrm{p}=0.0004$ ) on the constant work rate exercise test (WRET) and peak oxygen uptake increased (EPT, $\mathrm{p}=0.0028$; IPT, $\mathrm{p}=0.0072$ ). In the EPT group the following occurred: 1) soon after commencement of exercise with the constant WRET, the expiratory tidal volume $\left(V_{\mathrm{T}} \mathrm{ex}\right)$ increased, reducing dyspnoea; 2) $V_{\mathrm{T}}$ ex and mean expiratory flow increased and then prolonged expiration $(\mathrm{p}=0.0001)$ improved at peak exercise with the incremental exercise test (ET); and 3) St. George's Respiratory Questionnaire total, activity and impact scores were improved. In the IPT group, on both the constant WRET and incremental ET, breathing frequency increased, which led to greater exercise performance with effort dyspnoea.

Conclusions: This study showed the benefits of EPT/IPT on exercise performance. If the choice of managing COPD with EPT/IPT is appropriate, inexpensive EPT/IPT may become widespread as homebased training.

@ERSpublications

Expiratory or inspiratory pressure load training (EPT/IPT) based on the degree of prolonged expiration improves exercise performance in COPD patients. If managing COPD with EPT/IPT is chosen appropriately, it could become widespread as home-based training. https://bit.ly/ 2ZWutWq

Cite this article as: Miki $\mathrm{K}$, Tsujino $\mathrm{K}$, Miki M, et al. Managing COPD with expiratory or inspiratory pressure load training based on a prolonged expiration pattern. ERJ Open Res 2020; 6: 00041-2020 [https://doi.org/10.1183/23120541.00041-2020].

This article has supplementary material available from openres.ersjournals.com

Received: 25 Jan 2020 | Accepted after revision: 26 May 2020

Copyright $\odot$ ERS 2020. This article is open access and distributed under the terms of the Creative Commons Attribution Non-Commercial Licence 4.0. 


\section{Introduction}

Globally, COPD was the third leading cause of death in 2018 and 65 million people have moderate to severe COPD [1]. Furthermore, due to the prediction that global health spending will escalate from US\$10 trillion in 2015 to $\$ 20$ trillion in 2040 [2], countermeasures against COPD are thus pressing medical, economic and social issues.

Without reducing intolerable exertional dyspnoea, which is a hallmark, especially of the advanced stage of COPD, improving aerobic capacity and prognosis cannot be achieved. In COPD, exertional dyspnoea is recognised as correlating with dynamic air trapping and mechanical constraints on exertional tidal volume $\left(V_{\mathrm{T}}\right)$ expansion, which contribute the wasted ventilation. Given that humans develop exertional dyspnoea and a string of studies reported that exertional dyspnoea in COPD was associated with progressive mechanical constraints on $V_{\mathrm{T}}$ irrespective of the presence of dynamic hyperinflation [3-5], achieving the ability to expand $V_{\mathrm{T}}$ to obtain enough ventilation is extremely interesting as a treatment goal for COPD. Traditionally, as the stage of COPD advances, it has been recognised that, with rapid and shallow breathing, that is, with a lower $V_{\mathrm{T}}$, a higher breathing frequency $\left(f_{\mathrm{R}}\right)$ is required during exercise [6]. In fact, however, it is found that most such patients breathe slowly and shallowly with prolonged expiration during exercise. Both breathing patterns should be distinguished to select therapeutic approaches.

Inspiratory pressure load training (IPT), which has been extensively studied as inspiratory muscle training, has been recommended as a pulmonary rehabilitation (PR) programme. Several reviews suggested the effects of IPT as a stand-alone therapy, but, at least in advanced patients with COPD, no large studies have reported the adjunctive effects of IPT when added to PR [7-10]. In contrast, most reports related to expiratory pressure load training (EPT), known as expiratory muscle training, have investigated the expulsive effort, including sputum [11], perhaps because most EPT devices could provide a low-pressure load alone. Furthermore, WeINER et al. [12] reported that the effects of EPT added to IPT were not obtained in severe and very severe COPD. Therefore, the clue to investigate EPT has not been obtained in patients with COPD.

In our previous report, the hypothesis was formulated that EPT could be more effective in COPD patients when prolonged expiration patterns are confirmed by a decreased ratio of inspiratory time to total respiratory cycle time $\left(T_{\mathrm{I}} /\right.$ Ttot) during exercise, whereas IPT could be more effective when nonprolonged expiration patterns are confirmed by increased exertional $T_{\mathrm{I}} /$ Ttot [13]. To improve exercise performance of COPD patients with better exertional $T_{\mathrm{I}} / T$ tot, 1$)$ in the prolonged expiration patterns, a larger expiratory $V_{\mathrm{T}}\left(V_{\mathrm{T}} \mathrm{ex}\right)$ and a higher mean expiratory flow $\left(V_{\mathrm{T}}\right.$ ex/expiratory time $\left(T_{\mathrm{E}}\right)$ ) by EPT might be needed, although based on the Bernoulli principle, pursed-lip breathing needs the low expiratory flow and prolonged expiratory time to reduce airway collapse [14], and 2) in nonprolonged expiration patterns, the larger inspiratory $V_{\mathrm{T}}\left(V_{\mathrm{T}}\right.$ in) and higher mean inspiratory flow $\left(V_{\mathrm{T}}\right.$ in/inspiratory time $\left.\left(T_{\mathrm{I}}\right)\right)$ obtained by IPT might be needed.

Ghrelin has two forms including des-acyl ghrelin and acyl ghrelin; the latter is considered the major active form that has various physiological effects [15]. Edible medium-chain triglycerides (MCTs) change des-acyl ghrelin to acyl ghrelin [16]. Especially in patients with more advanced COPD, if the programme using IPT or EPT results in excessive training, and leads to a cachectic condition, edible MCTs might improve or maintain the patient's condition.

This open-label pilot study was conducted to prove the above hypothesis. The efficacy and safety of EPT or IPT with MCT administration were investigated in COPD patients, who were categorised according to their prolonged expiration pattern assessed by $T_{\mathrm{I}} /$ Ttot during cardiopulmonary exercise testing (CPET).

\section{Material and methods \\ Study design and patients}

Patients with moderate and very severe COPD were recruited in the National Hospital Organization Osaka Toneyama Medical Center from February 2018 to August 2019. Patients whose exertional $T_{\mathrm{I}} /$ Ttot decreased (resting $T_{\mathrm{I}} /$ tot $>$ peak $T_{\mathrm{I}} /$ Ttot) received 12-week EPT, while those whose exertional $T_{\mathrm{I}} /$ tot increased (resting $T_{\mathrm{I}} / T$ tot $\leqslant$ peak $T_{\mathrm{I}} /$ tot) received 12 -week IPT. Nutritional treatment with MCTs was given along with both training programmes.

This prospective study was conducted according to the Declaration of Helsinki and the Good Clinical Practice guidelines, was approved by the ethics committees of our centre (approval number, TNH-2018007) and was registered with the University Hospital Medical Information Network in Japan: https://upload.umin.ac.jp/cgi-open-bin/ctr_e/ctr_view.cgi?recptno=R000035288, number: UMIN000030937. All patients gave their written informed consent. 
The inclusion criteria were as follows: 1) moderate to very severe COPD based on spirometry: post-bronchodilator forced expiratory volume in $1 \mathrm{~s}\left(\mathrm{FEV}_{1}\right) /$ forced vital capacity $(\mathrm{FVC})$ ratio $<0.7$, and $\mathrm{FEV}_{1}$ percent predicted normal $<80 \%$ with modified British Medical Research Council: grade 1 and higher; 2 ) in a stable condition and able to tolerate CPET to ensure adequate evaluation; 3 ) aged between 40 and 85 years; and 4) provision of a signed agreement to participate in this study. Participants were excluded for any of the following reasons: 1) malignant tumours; 2) active infection; 3) severe heart disease; 4) asthma; 5) change in the drug regimen during this study; 6) receiving PR; 7) receiving oxygen therapy at rest; 8) on therapy for diabetes mellitus or with a glycated haemoglobin level $>7.0 \%$; and 9) judged by the physician to be inappropriate to participate in this study.

\section{Interventions}

Respiratory pressure load training

All patients received the training (30 times a set, two sets a day) for 12 weeks at home. The training load was started at $20 \%$ of the maximum expiratory pressure (MEP) or maximum inspiratory pressure (MIP) at baseline. If possible, the level was increased once every 2 weeks up to $50 \%$ of the maximum pressure. The EMST150 (Aspire Products, Atlanta, GA USA) and the POWERbreathe Medic plus (POWERbreathe International, Stratford Upon Avon, UK) were used for EPT and for IPT, respectively. Details are in the supplementary material.

\section{MCT administration}

Edible oil containing octanoic acid (C8) and decanoic acid (C10) (The Nisshin OilliO Group, Tokyo, Japan) was given at a dose of $2.5 \mathrm{~g}$ daily, orally, for 12 weeks.

\section{Evaluation}

Pulmonary function tests were done, as previously described [13, 17]. Symptom-limited exercise tests were conducted on an electrically-braked cycle ergometer (CV-1000SS, Lode, Groningen, The Netherlands), using a CPET system (Marquette CASE series T 2001, GE Healthcare, Tokyo, Japan; Aero monitor AE310S, Minato Medical Science Co., Ltd, Osaka, Japan), as previously described $[13,18]$. An incremental exercise test (ET), which consisted of 2-min increments to $10 \mathrm{~W}$, was performed until patient exhaustion without encouragement, especially during exercise. The constant work rate exercise test (WRET) was performed at $70 \%$ of the peak workload in the incremental ET before treatment. All patients were asked to maintain a cycle ergometer speed of about $60 \mathrm{rpm}$ while looking at the rpm meter. Minute ventilation $\left(V_{\mathrm{E}}^{\prime}\right)$, oxygen uptake $\left(V_{\mathrm{O}_{2}}^{\prime}\right)$, carbon dioxide output $\left(V_{\mathrm{CO}_{2}}^{\prime}\right), f_{\mathrm{R}}, V_{\mathrm{T}} \mathrm{in}, V_{\mathrm{T}} \mathrm{in} / T_{\mathrm{I}}, V_{\mathrm{T}} \mathrm{ex}, V_{\mathrm{T}} \mathrm{ex} / T_{\mathrm{E}}, T_{\mathrm{I}} /$ Ttot, physiologic dead space/tidal volume ratio $\left(V_{\mathrm{D}} / V_{\mathrm{T}}\right)$, and $\mathrm{O}_{2}$ pulse $\left(V_{\mathrm{O}_{2}}^{\prime}\right.$ /heart rate $\left.(\mathrm{HR})\right)$ were measured breath by breath and collected as 30-s average values at rest, during exercise at 2-min intervals and at the end of exercise. The anaerobic threshold (AT) was identified using the V-slope method and the nadir of the parameter $V_{E}^{\prime} / V_{\mathrm{O}_{2}}^{\prime}$ was measured during exercise. The severity of dyspnoea, intensity of leg fatigue and percutaneous oxygen saturation $\left(S_{\mathrm{pO}_{2}}\right)$ were evaluated by the modified Borg scale [19] at rest and during the last $15 \mathrm{~s}$ of each exercise stage and at the end of exercise. $V_{\mathrm{T}} \mathrm{in}-V_{\mathrm{T}}$ ex was evaluated as the volume of air remaining in the lung after expiration. Iso-time was defined as the highest equivalent exercise time for each subject among three evaluations. Dual-energy radiography absorptiometry and respiratory muscle strength evaluation by the MIP and MEP, respectively were performed as described previously $[13,20]$. The validated Japanese version of the St. George's Respiratory Questionnaire (SGRQ) was used as described previously $[16,21,22]$.

\section{Outcome measures}

\section{Efficacy}

The primary outcomes was the exertional change in $T_{\mathrm{I}} /$ Ttot. The secondary outcomes included: 1) the SGRQ score; 2) CPET parameters (peak $V_{\mathrm{O}_{2}}^{\prime}$, endurance time and dyspnoea intensity (Borg scale)); and 3) MEP and MIP.

\section{Safety}

All participants were included in the safety analyses.

\section{Sample size and statistical analysis}

At the time of the study design, no studies had investigated the efficacy of EPT or IPT in COPD patients based on the exertional prolongation of expiration by $T_{\mathrm{I}} / T$ tot. As this study protocol has never been previously performed, the sample size could not be calculated. This open-label pilot study evaluated at least 10 participants each for EPT and IPT. 
The effects of the intervention were examined twice (i.e. at Week 6 and Week 12) and were analysed in only each group. When no evaluations in the outcome were performed at Week 6 , the changes between at pretreatment and at Week 12 were analysed using the Wilcoxon signed rank test. The changes during the treatment time course (i.e. pretreatment, Week 6 and Week 12) were analysed using a linear mixed-effects model. The least squares means Tukey's honestly significant difference test was used for the comparison of each variable among the treatment points [23]. A p-value $<0.05$ was considered significant. Statistical analyses were performed using JMP software, version 11 (SAS Institute, Cary, NC, USA).

\section{Results}

Of the 22 enrolled patients, 21 completed this study protocol, because 1 patient dropped out after enrolment due to having his first episode of bronchial asthma during this study (figure 1). Eleven patients completed the EPT added to MCT administration (EPT group) protocol and 10 completed the IPT added to MCT administration (IPT group) protocol, whose data were used to ensure efficacy using a per-protocol analysis. All patients enrolled in the EPT group had severe to very severe airway obstruction with reduced exercise tolerance and seven patients had a history of PR. The EPT group had a higher frequency of hospitalisation than the IPT group. Table 1 shows the patients' baseline characteristics.

\section{EPT and IPT progression}

The ratio of the attained pressure after the 12 -week programme to the target pressure was $78 \% \pm 19 \%$ in the EPT group and $95 \% \pm 16 \%$ in the IPT group.

\section{Evaluation in the EPT group}

Only MEP increased during the whole EPT treatment course (table 2).

Changes at peak exercise in the incremental ET for 12 weeks are shown in figure 2a-e and table 2. The $V_{\mathrm{O}_{2}}^{\prime}(\mathrm{p}=0.0028), V_{\mathrm{T}} \mathrm{ex}(\mathrm{p}=0.0418), V_{\mathrm{T}} \mathrm{ex} / T_{\mathrm{E}}(\mathrm{p}=0.0025), V_{\mathrm{T}} \mathrm{in}-V_{\mathrm{T}} \mathrm{ex}(\mathrm{p}=0.0339)$ and $T_{\mathrm{I}} / T$ tot $($ peak-rest $)$ $(\mathrm{p}=0.0001)$ improved significantly during the whole EPT treatment course. When comparing each parameter among the treatment points, significant improvements compared with pretreatment, which were confirmed at both 6 and 12 weeks, were observed in $V_{\mathrm{T}} \mathrm{ex} / T_{\mathrm{E}}$ and $T_{\mathrm{I}} /$ Ttot (peak-rest).

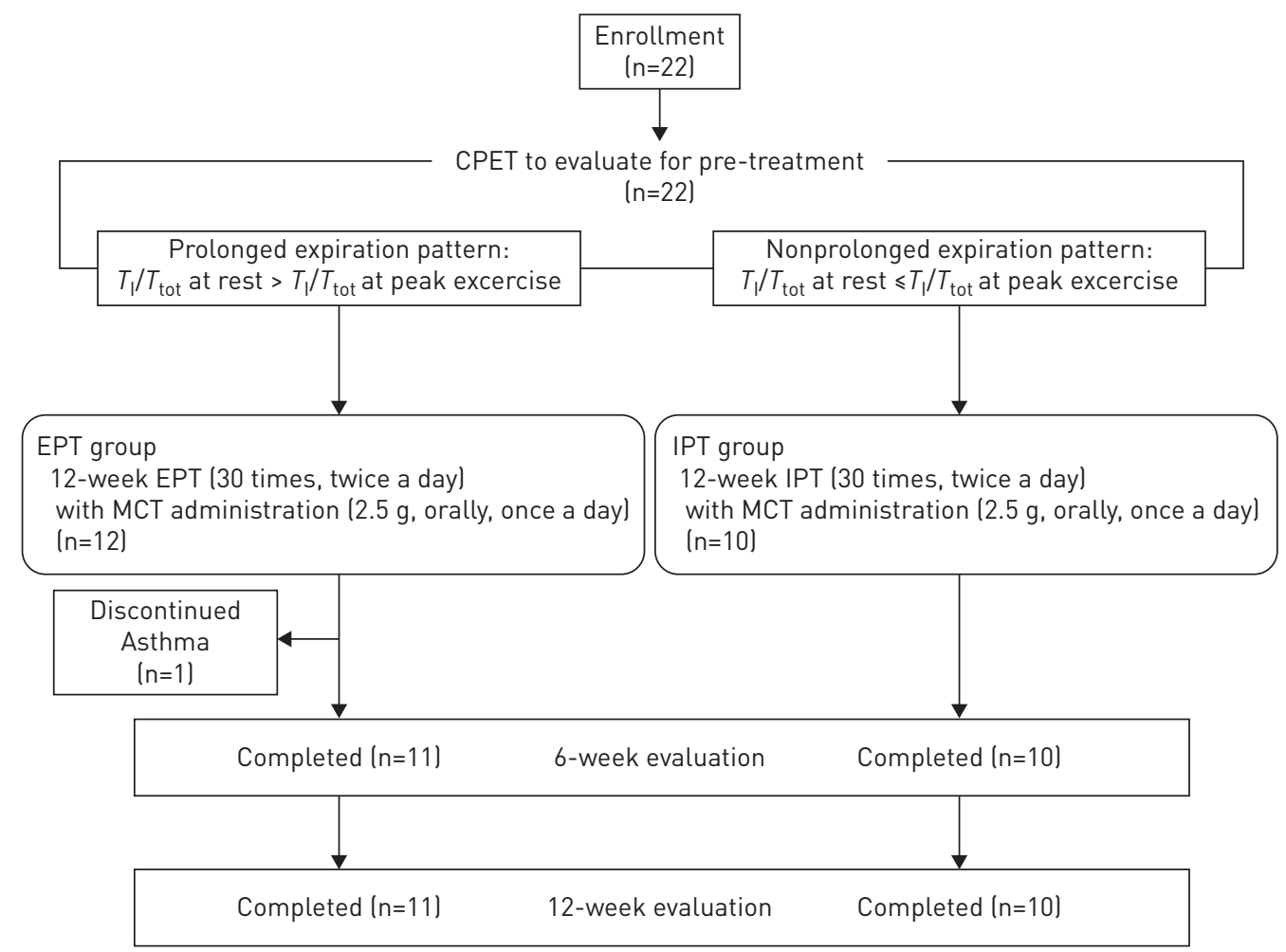

FIGURE 1 Trial profile. CPET: cardiopulmonary exercise testing; EPT: expiratory pressure load training; IPT: inspiratory pressure load training; MCT: medium-chain triglycerides; $T_{1} /$ Ttot: the ratio of inspiratory time to total respiratory cycle time. 


\section{TABLE 1 Baseline characteristics of the patients $(n=21)$}

\begin{tabular}{|c|c|c|c|}
\hline & EPT group $(n=11)$ & IPT group $(n=10)$ & p-value \\
\hline Age years & $67.3 \pm 5.3$ & $73.4 \pm 4.6$ & 0.1111 \\
\hline Sex male/female $n$ & $10 / 1$ & $10 / 0$ & 0.3286 \\
\hline $\mathrm{BMI} \mathrm{kg} \cdot \mathrm{m}^{-2}$ & $20.7 \pm 2.5$ & $20.8 \pm 3.5$ & 1.0000 \\
\hline Cigarette smoking pack-years & $62.0 \pm 26.9$ & $70.2 \pm 29.0$ & 0.7245 \\
\hline GOLD, II/III/IV n & $0 / 7 / 4$ & $4 / 5 / 1$ & 0.0346 \\
\hline \multicolumn{4}{|l|}{ Pulmonary function test } \\
\hline $\mathrm{FEV}_{1} \mathrm{~L}$ & $0.87 \pm 0.19$ & $1.18 \pm 0.40$ & 0.1486 \\
\hline $\mathrm{FEV}_{1} \%$ predicted & $32.8 \pm 7.0$ & $45.1 \pm 14.2$ & 0.0448 \\
\hline $\mathrm{FEV}_{1} / \mathrm{FVC} \%$ & $33.9 \pm 6.0$ & $38.9 \pm 6.5$ & 0.1130 \\
\hline VC L & $2.75 \pm 0.56$ & $3.14 \pm 0.80$ & 0.2048 \\
\hline VC \% predicted & $84.8 \pm 12.3$ & $98.0 \pm 22.8$ & 0.0980 \\
\hline \multicolumn{4}{|l|}{ Incremental work rate exercise testing } \\
\hline Peak $V_{\mathrm{O}_{2}}^{\prime} \mathrm{mL} \cdot \mathrm{min}^{-1} \cdot \mathrm{kg}^{-1}$ & $10.7 \pm 2.7$ & $11.6 \pm 3.4$ & 0.5968 \\
\hline AT $\mathrm{mL} \cdot \mathrm{min}^{-1} \cdot \mathrm{kg}^{-1}$ & $8.8 \pm 1.9$ & $10.8 \pm 2.0$ & 0.0736 \\
\hline \multicolumn{4}{|l|}{ Medications $\mathrm{n}$} \\
\hline LAMA/LABA/ICS & $10 / 11 / 8$ & $10 / 10 / 4$ & 0.6321 \\
\hline Triple inhalation therapy & 8 & 4 & 0.1301 \\
\hline Long-term oxygen therapy ${ }^{\#}$ & 3 & 2 & 0.6959 \\
\hline History of pulmonary rehabilitation & 7 & 4 & 0.2787 \\
\hline History of hospitalisation & 8 & 2 & 0.0157 \\
\hline \multicolumn{4}{|c|}{$\begin{array}{l}\text { Data are presented as mean } \pm \text { SD, unless otherwise stated. EPT: expiratory pressure load training; IPT: } \\
\text { inspiratory pressure load training; BMI: body mass index; GOLD: global initiative for chronic obstructive } \\
\text { lung disease; FEV } V_{1} \text { : forced expiratory volume in } 1 \mathrm{~s} \text {; FVC: forced vital capacity; VC: vital capacity; AT: } \\
\text { anaerobic threshold; } V_{O_{2}} \text { : oxygen uptake; LABA: long-acting } \beta_{2} \text {-agonist; LAMA: long-acting muscarinic } \\
\text { antagonist; ICS: inhaled corticosteroids. Triple inhalation: a combination of LAMA, LABA and ICS. } \\
\text { Medications are presented separately. ATs were not detectable in four patients in the EPT group, and in } \\
\text { three patients in the IPT group. History of hospitalisation owing to a deteriorated condition in relation to } \\
\text { COPD was investigated. " : All patients used long-term oxygen therapy only on exertion. }\end{array}$} \\
\hline
\end{tabular}

Changes at the limit of tolerance and during exercise on the constant WRET for 12 weeks are shown in figure $3 \mathrm{a}-\mathrm{c}$ and tables 3 and 4 . The increased endurance time was highly significant $(\mathrm{p}<0.0001)$ and the significance was evident already from 6 weeks. Increased leg fatigue seemed to stop the prolonged exercise obtained from EPT. The exertional dyspnoea was reduced significantly at iso-time (figure $3 \mathrm{~b}$ and table 4). The $V_{\mathrm{T}}$ ex increased from the early exercise phase (figure $3 \mathrm{c}$ ) and the $f_{\mathrm{R}}$ was reduced at iso-time (table 4 ).

All SGRQ scores except for the SGRQ symptoms domain improved (table 5). Pulmonary functions and body weight were unchanged (table 5 ).

\section{Evaluation in the IPT group}

During the whole IPT treatment course: 1) only MIP increased; 2) at peak exercise in the incremental ET during 12 weeks, $V_{\mathrm{O}_{2}}^{\prime}(\mathrm{p}=0.0072)$ and $V_{\mathrm{E}}^{\prime}(\mathrm{p}=0.0147)$ improved and exertional dyspnoea worsened with increased $f_{\mathrm{R}}$ and unchanged $V_{\mathrm{T}} \mathrm{ex}$ (figure $2 \mathrm{f}-\mathrm{j}$ and table 2 ); and 3 ) on the constant WRET, the increased endurance time was highly significant $(\mathrm{p}=0.0004)$. At the limit of tolerance, however, the $f_{\mathrm{R}}$ and $V_{\mathrm{D}} / V_{\mathrm{T}}$ were increased with worsened exertional dyspnoea and leg fatigue (figure 3e,f and table 3). All SGRQ scores, pulmonary functions and body weight did not improve (table 5).

\section{Safety}

Loose stools were observed in 2 of the 22 enrolled patients. No treatment-related serious events such as a pneumothorax were reported.

\section{Discussion}

The main findings of this study were as follows. First, 12-week EPT with MCT administration: 1) improved quality of life (QOL) based on the SGRQ scores, the peak $V^{\prime}{ }_{\mathrm{O}_{2}}$, and the endurance time; 2) increased $V_{\mathrm{T}}$ ex and $V_{\mathrm{T}} \mathrm{ex} / \mathrm{T}_{\mathrm{E}}$ and decreased $V_{\mathrm{T}} \mathrm{in}-V_{\mathrm{T}}$ ex at peak exercise on the incremental ET, which improved prolonged expiration (primary outcome); and 3) increased $V_{\mathrm{T}}$ ex from the commencement of the exercise on the constant WRET and then the exertional dyspnoea was reduced at iso-time. Second, 12-week IPT with MCT administration: 1) did not improve the SGRQ scores, but it improved peak $V_{\mathrm{O}_{2}}^{\prime}$ 
TABLE 2 Changes at peak exercise in the incremental exercise parameters and resting respiratory muscle strength after respiratory pressure load training

\begin{tabular}{|c|c|c|c|c|c|c|c|c|}
\hline & \multicolumn{3}{|c|}{ EPT group (n=11) } & p-value & \multicolumn{3}{|c|}{ IPT group $(n=10)$} & p-value \\
\hline$V_{\mathrm{O}_{2}}^{\prime} \mathrm{mL} \cdot \min ^{-1}$ & $613.9 \pm 199.2$ & $639.5 \pm 223.0$ & $670.2 \pm 233.2^{* *}$ & 0.0025 & $646.0 \pm 220.9$ & $657.6 \pm 243.7$ & $694.5 \pm 255.7^{* *} . \#$ & 0.0091 \\
\hline$V_{\mathrm{O}_{2}}^{\prime} \mathrm{mL} \cdot \mathrm{min}^{-1} \cdot \mathrm{kg}^{-1}$ & $10.7 \pm 2.7$ & $11.2 \pm 3.1$ & $11.6 \pm 3.1^{* *}$ & 0.0028 & $11.6 \pm 3.4$ & $11.7 \pm 3.7$ & $12.4 \pm 3.9^{*, \#}$ & 0.0072 \\
\hline$V_{E}^{\prime} L \cdot \min ^{-1}$ & $27.9 \pm 7.0$ & $29.1 \pm 7.3$ & $30.1 \pm 8.3^{* * *}$ & 0.0006 & $30.1 \pm 8.5$ & $30.8 \pm 9.8$ & $32.3 \pm 11.0^{*}$ & 0.0147 \\
\hline$V_{E}^{\prime} / V_{\mathrm{O}_{2}}^{\prime}$ & $46.5 \pm 4.8$ & $46.7 \pm 6.3$ & $46.1 \pm 5.6$ & 0.7966 & $48.3 \pm 8.4$ & $48.8 \pm 10.5$ & $47.7 \pm 8.3$ & 0.4216 \\
\hline$V_{\mathrm{E}}^{\prime} / V_{\mathrm{CO}_{2}}^{\prime}$ & $47.0 \pm 5.7$ & $46.8 \pm 6.8$ & $46.2 \pm 6.6$ & 0.7626 & $48.6 \pm 9.7$ & $48.4 \pm 11.0$ & $47.9 \pm 9.6$ & 0.7709 \\
\hline$V_{\mathrm{D}} / V_{\mathrm{T}}$ & $0.42 \pm 0.04$ & $0.41 \pm 0.04$ & $0.41 \pm 0.04$ & 0.2974 & $0.41 \pm 0.05$ & $0.41 \pm 0.05$ & $0.41 \pm 0.05$ & 0.8071 \\
\hline HR beats. $\min ^{-1}$ & $119 \pm 13$ & $120 \pm 12$ & $125 \pm 12^{*}$ & 0.0142 & $113 \pm 15$ & $117 \pm 19$ & $117 \pm 17$ & 0.4643 \\
\hline $\mathrm{O}_{2}$ pulse $\mathrm{mL} \cdot$ beats $^{-1}$ & $5.1 \pm 1.3$ & $5.3 \pm 1.5$ & $5.3 \pm 1.5$ & 0.3072 & $5.8 \pm 1.7$ & $5.5 \pm 1.7$ & $5.6 \pm 1.7$ & 0.2675 \\
\hline $\mathrm{S}_{\mathrm{pO}_{2}} \%$ & $89 \pm 5$ & $89 \pm 4$ & $88 \pm 4$ & 0.1970 & $91 \pm 4$ & $89 \pm 4$ & $91 \pm 4$ & 0.1433 \\
\hline$V_{\mathrm{T}} \mathrm{ex} / T_{\mathrm{E}} \mathrm{mL} \cdot \mathrm{s}^{-1}$ & $651 \pm 169$ & $706 \pm 202^{*}$ & $728 \pm 217^{* *}$ & 0.0025 & $820 \pm 276$ & $847 \pm 334$ & $884 \pm 365^{*}$ & 0.0347 \\
\hline$V_{\mathrm{T}}$ in $-V_{\mathrm{T}}$ ex mL & $25 \pm 44$ & $5 \pm 19$ & $1 \pm 26^{*}$ & 0.0339 & $2 \pm 26$ & $0 \pm 44$ & $-15 \pm 40$ & 0.2506 \\
\hline$T_{1} / T$ tot peak - rest & $-0.04 \pm 0.06$ & $-0.00 \pm 0.02^{* * *}$ & $-0.01 \pm 0.03^{* *}$ & 0.0001 & $0.04 \pm 0.02$ & $0.02 \pm 0.04$ & $0.02 \pm 0.03$ & 0.5356 \\
\hline MEP $\mathrm{cmH}_{2} \mathrm{O}$ & $125.4 \pm 24.7$ & $149.2 \pm 33.0 *$ & $153.4 \pm 32.4^{* *}$ & 0.0047 & $114.9 \pm 45.4$ & $110.5 \pm 40.7$ & $117.9 \pm 47.1$ & 0.5943 \\
\hline MIP $\mathrm{cmH}_{2} \mathrm{O}$ & $77.9 \pm 19.3$ & $81.2 \pm 20.2$ & $85.9 \pm 21.8$ & 0.1530 & $67.9 \pm 17.2$ & $74.2 \pm 17.4$ & $78.0 \pm 19.0^{*}$ & 0.0217 \\
\hline
\end{tabular}

Data are presented as mean \pm SD. EPT: expiratory pressure load training; IPT: inspiratory pressure load training; $V^{\prime}{ }_{O_{2}}$ : oxygen uptake; $V^{\prime}{ }_{E}:$ minute ventilation; $V_{\mathrm{T}}$ ex: expiratory tidal volume; $f_{\mathrm{R}}$ : breathing frequency; $V^{\prime} \mathrm{CO}_{2}$ : carbon dioxide output; $V_{\mathrm{D}} / V_{\mathrm{T}}$ : physiologic dead space/tidal volume ratio; HR: heart rate; $\mathrm{O}_{2}$ pulse: $V^{\prime}{ }_{\mathrm{O}_{2}} / \mathrm{HR} ; \mathrm{S}_{\mathrm{pO}_{2}}$ : percutaneous oxygen saturation; $T_{1}$ : inspiratory time; $V_{\mathrm{T}}$ in: inspiratory tidal volume; $T_{\mathrm{E}}$ : expiratory time; $T_{1} /$ Ttot: inspiratory duty cycle; MEP: maximal expiratory pressure; MIP: maximal inspiratory pressure. ${ }^{*}: p<0.05 ;{ }^{* *}: p<0.01 ;{ }^{* * *}: p<0.001$, by the least squares means Tukey honestly significant difference, compared with the pretreatment; ${ }^{\#}: p<0.05$, by the least squares means Tukey's honestly significant difference, compared with the values after 6 weeks of IPT.

and endurance time; and 2) increased the $f_{\mathrm{R}}$ with increased exertional dyspnoea and without a change of exertional $T_{\mathrm{I}} /$ Ttot (primary outcome) on the incremental ET and the constant WRET.

It has been considered that training patients with COPD to breathe slowly and deeply for rapid and shallow breathing during exercise, which leads to dynamic hyperinflation, is useful to reduce exertional dyspnoea [6]. However, slow and shallow breathing, instead of rapid and shallow breathing, unexpectedly leads to exertional dyspnoea to stop exercise. NeDER et al. [24] reported that, in a study of mild to end-stage COPD including healthy controls, the peak $f_{\mathrm{R}}$ with the incremental ET decreased to stop exercise as the disease stage advanced. Also, in the present study, reduced $f_{\mathrm{R}}$ accompanied by reduced $V_{\mathrm{T}} \mathrm{ex}$ was confirmed in both groups pretreatment. Moreover, in the EPT group, patients with a more advanced stage than those in the IPT group breathed slowly and shallowly with the exertional prolongation of expiration (table 2). Training such patients using, for example, pursed-lip breathing, would be less useful, because slower breathing might be impossible and ineffective to obtain enough ventilation. Therefore, countermeasures against slow and shallow breathing with prolonged expiration of COPD have largely been effectively overlooked.

On the EPT, the increase in endurance time, measured with the constant WRET, was large. The 5.7-min increase with reduced exertional dyspnoea was long enough, given that: 1) the clinical meaningful difference is $100 \mathrm{~s}$ [18]; and 2) patients with severe and very severe COPD and severely reduced exercise tolerance, who had already taken most available treatments including PR enrolment (table 1), all obtained a prolonged endurance time (figure 3a). The adequate aerobic capacity with the improvement in QOL is rewarding. In the EPT group, all treatment differences in the SGRQ total, impact and total scores were more than seven units, which was higher than the four units accepted as clinically relevant [22]. MотA et al. [25] reported that, in severe COPD, 5-week EPT improved the SGRQ scores but not ventilatory variables, including peak $V^{\prime} \mathrm{O}_{2}$. Their findings are consistent with our results in which the unchanged peak $V^{\prime} \mathrm{O}_{2}$ was obtained after 6 weeks in the EPT group, suggesting that the duration of EPT needs to be more than 6 weeks. Of note, in the present study, it was demonstrated that the increase of $V_{\mathrm{T}} \mathrm{ex} / T_{\mathrm{E}}$ and the 

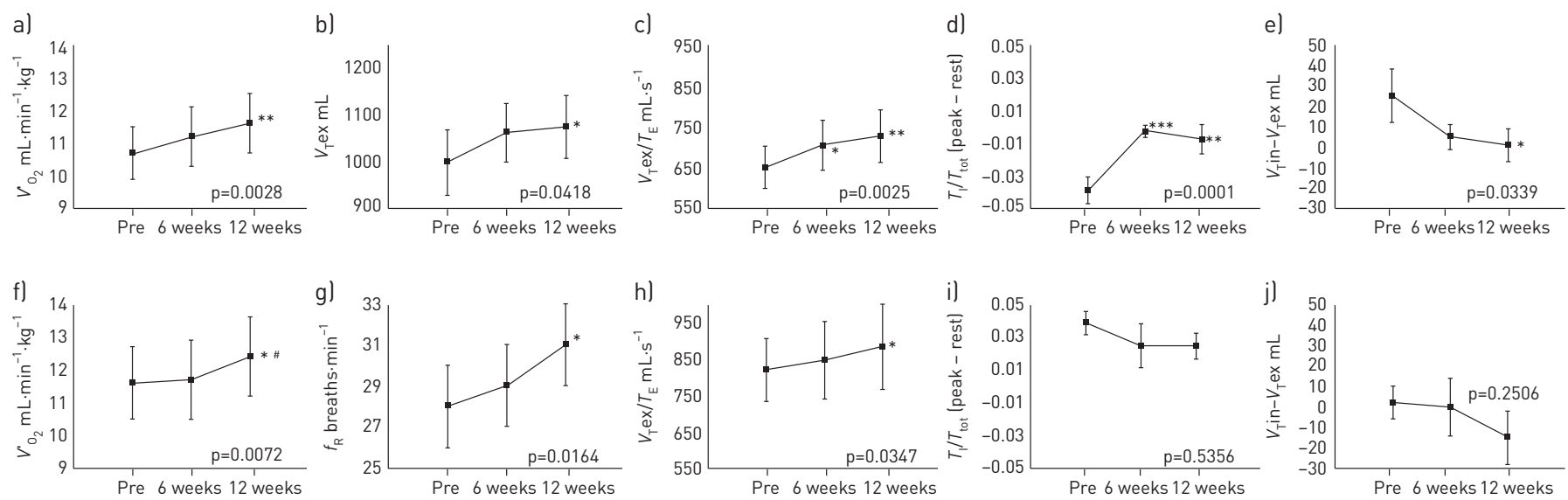

FIGURE 2 Changes at peak exercise on the incremental exercise testing after expiratory pressure load training (EPT, panels a-e) or inspiratory pressure load training (IPT, panels $\mathrm{f}-\mathrm{j}$ ). $T_{\mathrm{E}}$ : expiratory time; $T_{1} / T$ tot: the ratio of inspiratory time to total respiratory cycle time; $V^{\prime}{ }_{\mathrm{O}_{2}}$ : oxygen uptake; $V_{\mathrm{T}}$ ex: expiratory tidal volume; $V_{\mathrm{T}} \mathrm{ex} / T_{\mathrm{E}}$ : mean expiratory flow; $V_{\mathrm{T}}$ in: inspiratory tidal volume; $f_{\mathrm{R}}$ : breathing frequency. By a linear mixed-effect model: $p$-value. By the least squares means Tukey's honestly significant test: ${ }^{*}: p<0.05,{ }^{* *}: p<0.01,{ }^{* * *}: p<0.001$, compared with the pretreatment, ${ }^{\#}: \mathrm{p}<0.05$, compared with after 6 weeks of treatment.

improved prolonged expiration from baseline were confirmed from 6 weeks, but the increase of $V_{\mathrm{T}}$ ex was not significant (table 2 and figure 2). These responses might mean that after EPT, even if the $T_{\mathrm{E}}$ was markedly reduced, enough expiratory volume was obtained without airway collapse. Furthermore, at 12 weeks, a larger $V_{\mathrm{T}}$ ex or higher $V_{\mathrm{T}} \mathrm{ex} / T_{\mathrm{E}}$ obtained from EPT improved exercise performance and exertional dyspnoea in advanced COPD. EPT might thus prevent dynamic wasted ventilation, as the
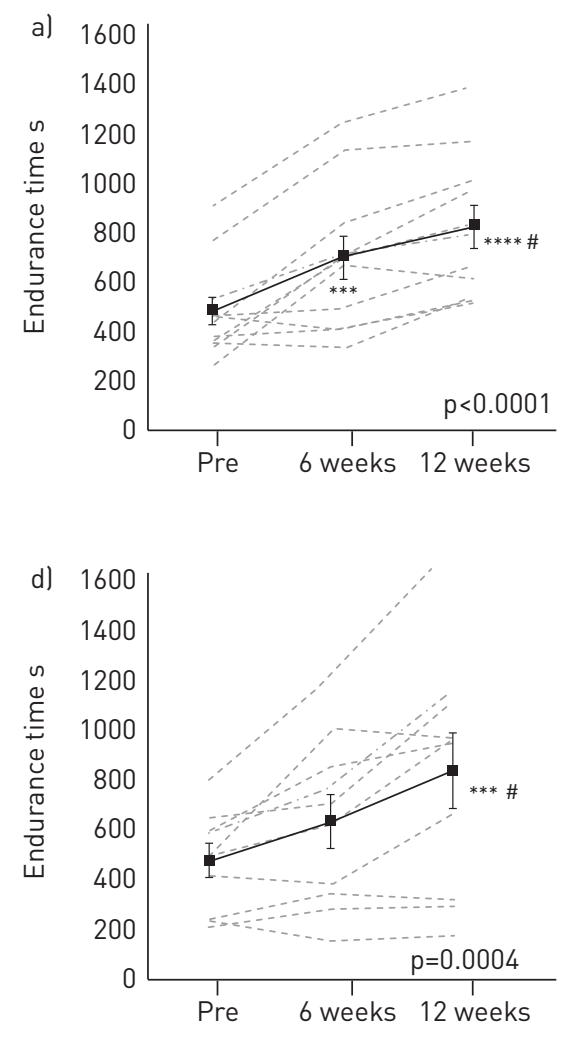
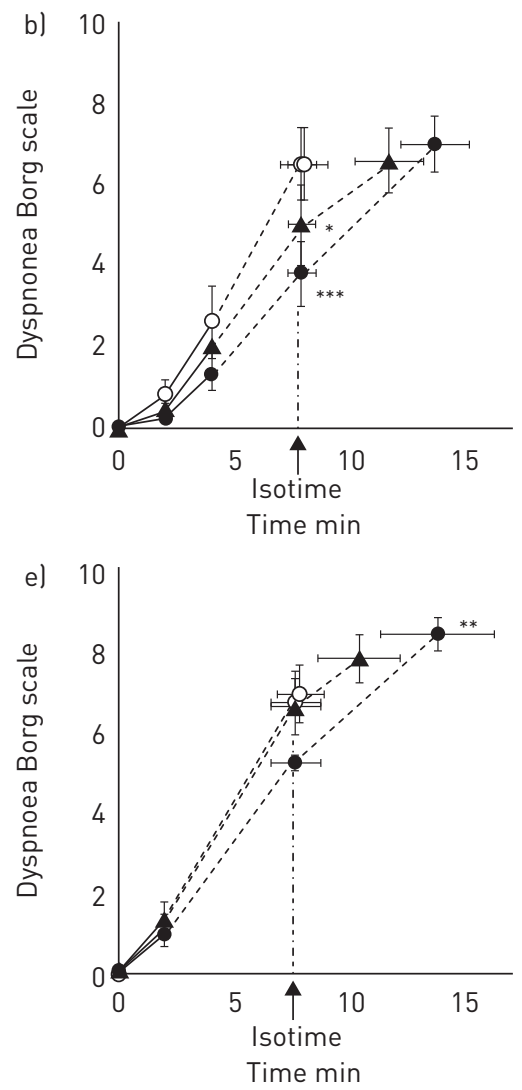
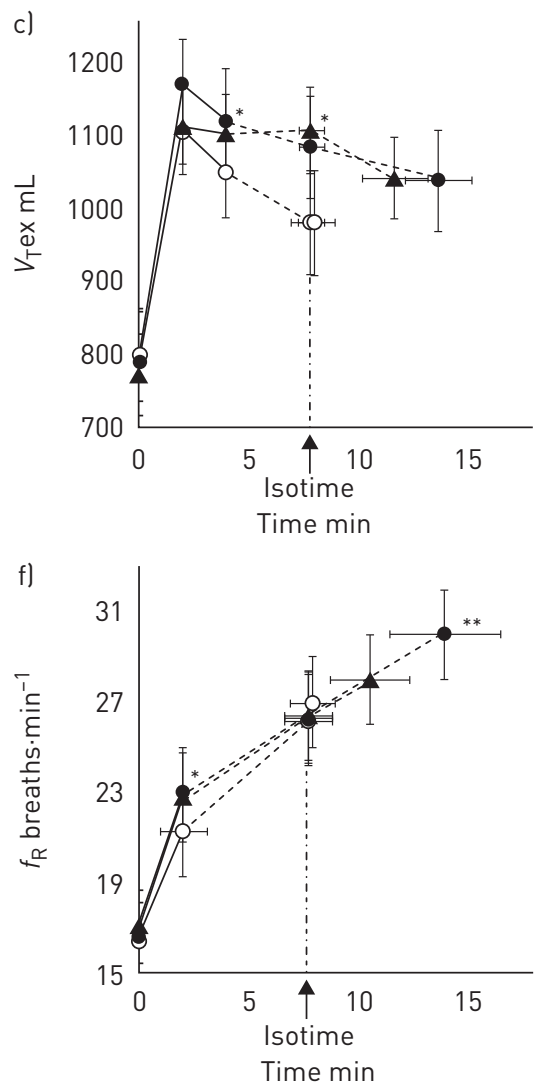

FIGURE 3 Changes at the limit of tolerance and during exercise on the constant work rate exercise testing after expiratory pressure load training

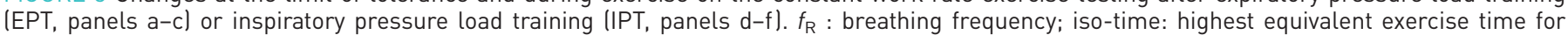
each subject among three evaluations; $V_{T}$ ex: expiratory tidal volume. Open circle: pretreatment; closed triangle: after 6 weeks of treatment; closed

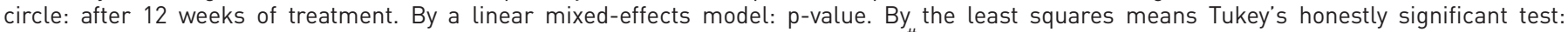
${ }^{*}: \mathrm{p}<0.05,{ }^{* *}: \mathrm{p}<0.01,{ }^{* * *}: \mathrm{p}<0.001,{ }^{* * *}: \mathrm{p}<0.0001$, compared with the pretreatment; ${ }^{*}$ : $<<0.05$, compared with after 6 weeks of treatment. 
TABLE 3 Changes at the limit of tolerance in the constant work rate exercise parameters after respiratory pressure load training

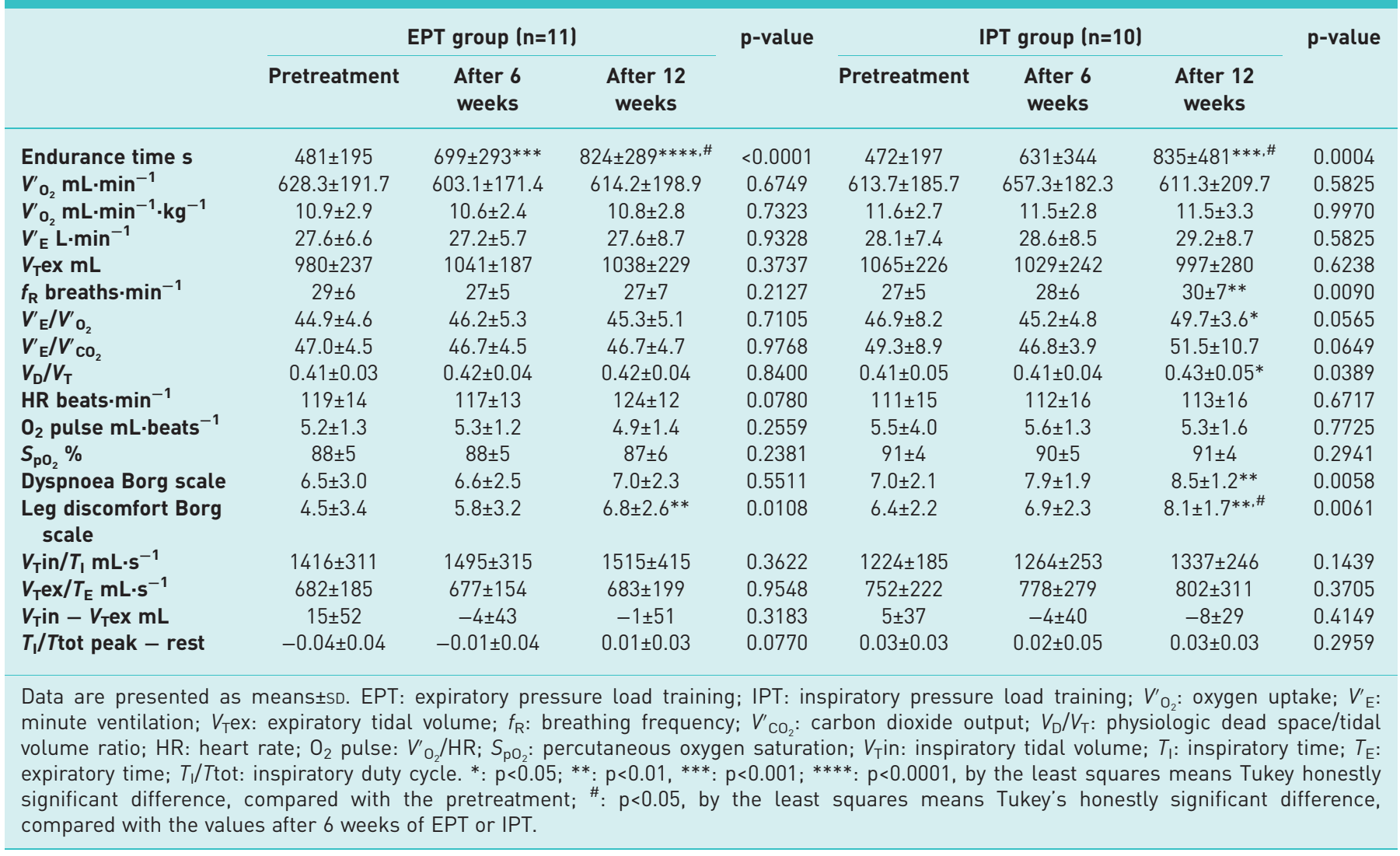

reduced air remaining in the lung after expiration, that is $V_{\mathrm{T}} \mathrm{in}-V_{\mathrm{T}} \mathrm{ex}$, on the incremental ET was confirmed. It is unclear, however, where the site of action for EPT is and how EPT affects it. Laryngeal narrowing occurs in humans during the expiratory phase, and as the stage of COPD advances, the larynx narrows more during exercise, and this response is related to longer prolonged expiration, lower $V_{\mathrm{T}}$ ex, and lower peak $V_{\mathrm{O}_{2}}^{\prime}[26,27]$. As one of the possible mechanisms of EPT, EPT might promote laryngeal widening by affecting the laryngeal abductors [27], which could prevent the vocal cords with central airways from collapsing during exercise. In the future, observation of glottic function by laryngoscopy during exercise might prove the above possible mechanism.

In IPT, the increased ventilation resulting from rapid $f_{\mathrm{R}}$ leads to increased aerobic capacity and endurance time. On the constant WRET, figure $3 \mathrm{f}$ shows that the $f_{\mathrm{R}}$ levels were similar at iso-time among all evaluation points, and the slope of $f_{\mathrm{R}}$ to the endurance time was not changed after IPT. In addition, tables 3 and 4 show that, after IPT, all ventilatory variables were not changed at iso-time, but the increased $f_{\mathrm{R}}$-related variables $\left(V_{\mathrm{D}} / V_{\mathrm{T}}, V^{\prime}{ }_{\mathrm{E}} / V^{\prime} \mathrm{O}_{2}\right.$ and dyspnoea) were increased at the limit of tolerance. These findings suggest that IPT enabled exercise by forcing greater additional effort than pretreatment levels using the rapid $f_{\mathrm{R}}$. In fact, after IPT, only one patient with the highest $\% \mathrm{FEV}_{1}$ among the IPT group could exercise longer, reducing the $f_{\mathrm{R}}$ with the higher $V_{\mathrm{T}}$ ex. If wasted ventilation does not occur, the rapid $f_{\mathrm{R}}$ may be attractive for getting enough ventilation, because healthy subjects can reach a rapid $f_{\mathrm{R}}$ and patients with advanced COPD cannot reach it to stop exercise [24]. After IPT, three patients could not achieve enough prolongation of endurance time (figure $3 \mathrm{~d}$ ) due to the increased $f_{\mathrm{R}}$-related wasted ventilation. These findings suggested that IPT is not indicated for patients with wasted ventilation. WEINER et al. [12] reported that adding IPT to EPT did not show additional effects, although the effect of EPT alone was confirmed. The forcing effort owing to the increased $f_{\mathrm{R}}$ by IPT might hide the effect of EPT in advanced COPD.

Weight and $\mathrm{O}_{2}$ pulse, changes in both of which were attributed to the effect of MCT administration [16], were unchanged in both groups. There was, therefore, little effect of MCT administration.

This study had some limitations. First, further studies are necessary to investigate whether patients without exertional prolongation of expiration are appropriate for EPT. Second, the EMST150 is generally used by 
TABLE 4 Changes at iso-time in the constant work rate exercise parameters after respiratory pressure load training

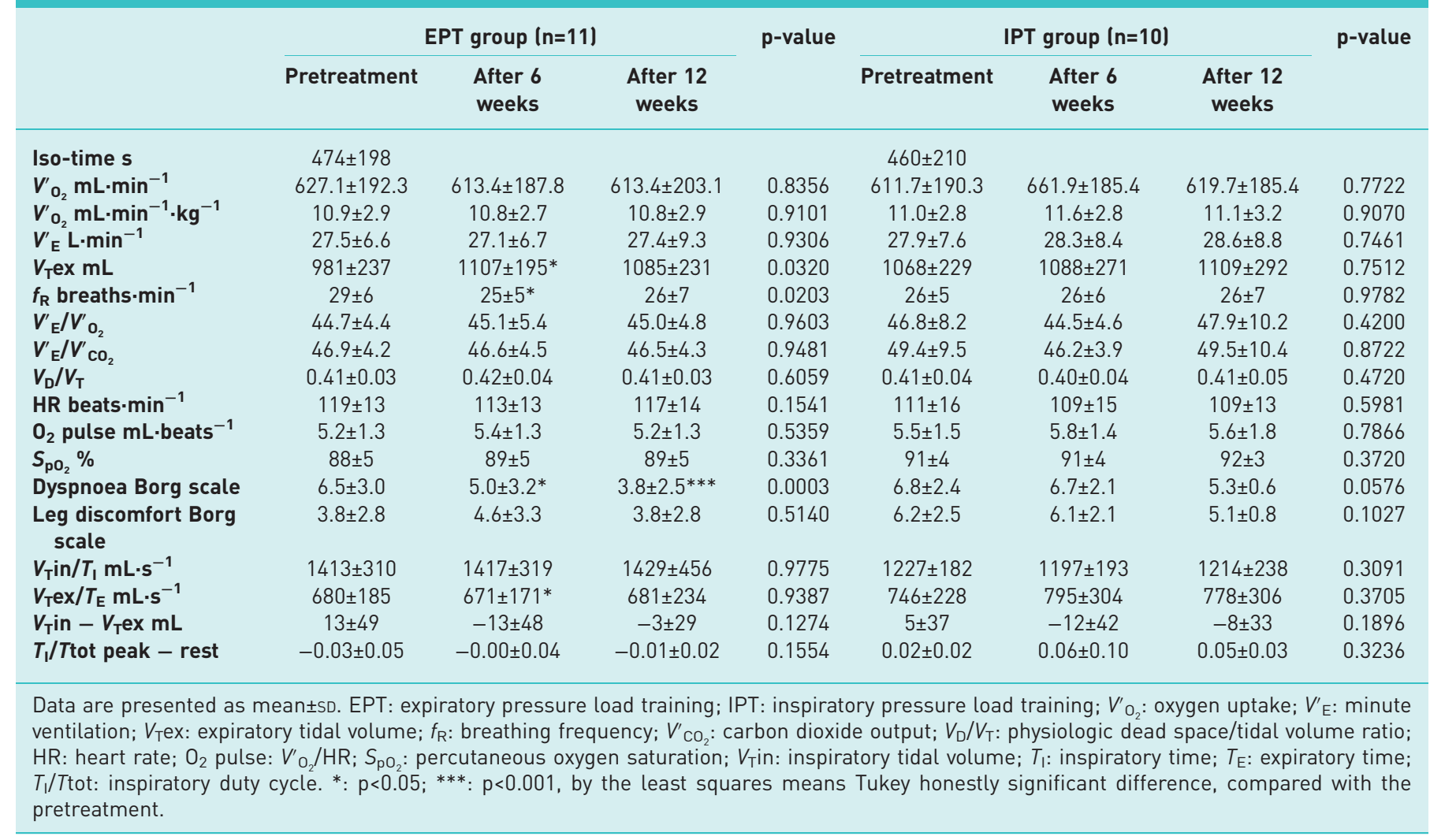

singers and athletes. Managing to get a suitable device for EPT, which can accurately select the specified pressure for each COPD patient, is necessary. Third, the profoundly improved exercise performance provided new problems: leg discomfort appeared as an exercise limitation, which required exercise training

TABLE 5 Changes in the resting parameters after respiratory pressure load training

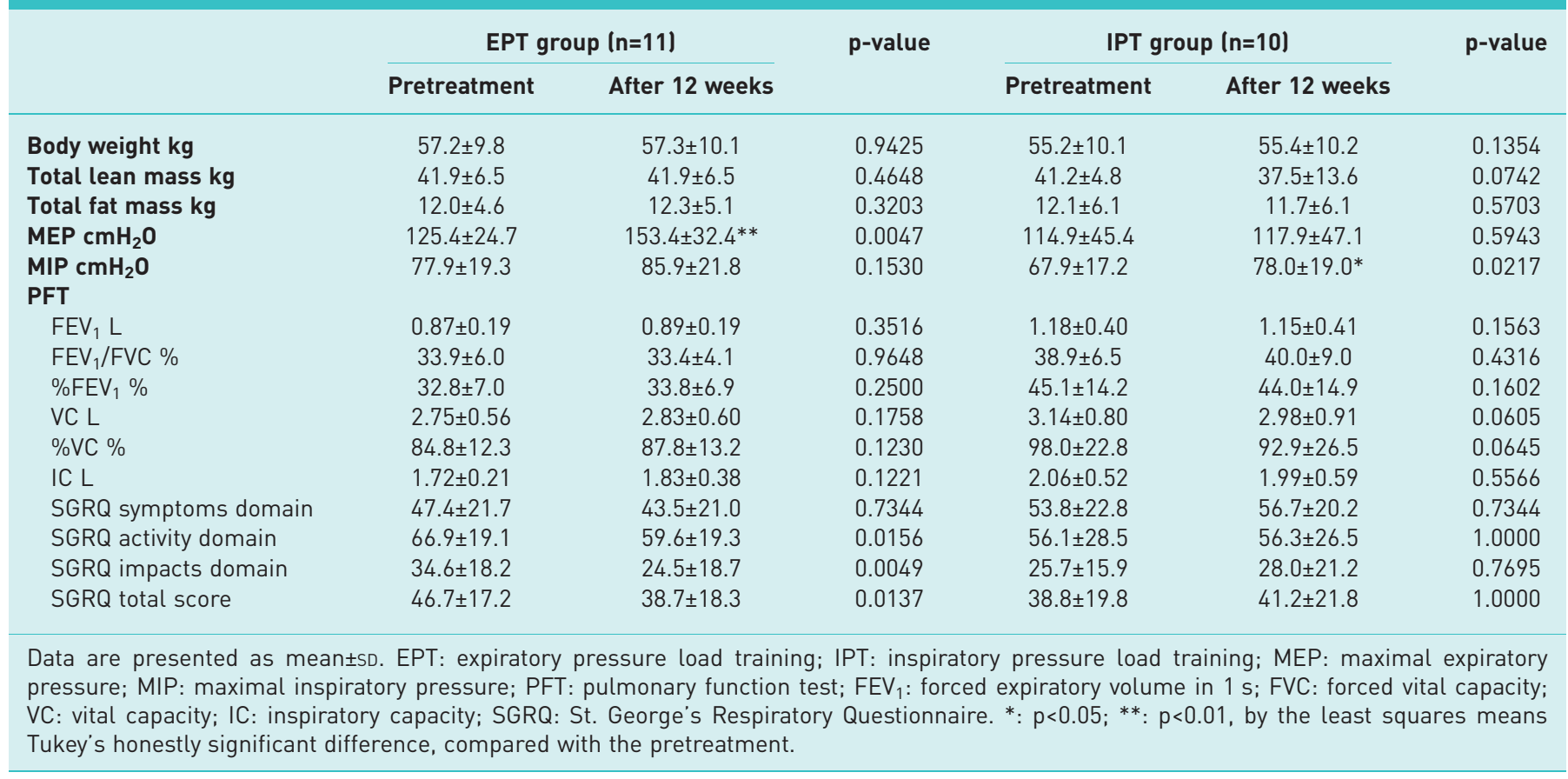


for the legs. Fourth, the increase of MIP after IPT was low, which might be related to insufficient IPT. Although the increase in endurance time, measured with constant WRET, was large enough with IPT, a suitable regimen for IPT is necessary. Given the useful results obtained, future studies, including randomised controlled trials, should be conducted to develop EPT/IPT.

In conclusion, this study showed the benefits of 12-week EPT and IPT based on the exertional prolonged expiration on aerobic capacity and endurance time in COPD patients. After EPT, a larger $V_{\mathrm{T}}$ ex and higher $V_{\mathrm{T}} \mathrm{ex} / T_{\mathrm{E}}$ reduced the air remaining in the lung after expiration and improved exertional prolongation of expiration, reducing exertional dyspnoea. After IPT, the increased ventilation from the increased $f_{\mathrm{R}}$ resulted in unchanged breathing timing and worsening of dyspnoea during exercise. Given that both EPT and IPT are inexpensive and can easily be handled by elderly patients, if managing COPD with EPT/IPT is selected appropriately, it could become widespread as home-based training.

Acknowledgements: The authors thank E. Oda (AC Medical, Tokyo, Japan) for his assistance in statistical analysis. This work was previously presented at the ERS International Congress 2019, Madrid, Spain.

Author contributions: All authors contributed substantially to this article. K. Miki conceived and designed the study, performed the experiments, analysed the data and wrote the manuscript. K. Tsujino and M. Miki conceived and designed the study, performed the experiments and analysed the data. K. Yoshimura, H. Kagawa, Y. Oshitani, K. Fukushima, T. Matsuki, Y. Yamamoto and H. Kida performed the experiments and analysed the data. Each author approved the submission of this manuscript for publication.

Conflict of interest: None declared.

Support statement: This study was supported by a Grant-in-Aid for Clinical Research from the National Hospital Organization. Funding information for this article has been deposited with the Crossref Funder Registry.

\section{References}

1 World Health Organization. The top 10 causes of death. www.who.int/news-room/fact-sheets/detail/the-top-10causes-of-death Date last accessed: November 19, 2019. Date last updated: May 24, 2018.

2 Global Burden of Disease Health Financing Collaborator Network. Trends in future health financing and coverage: future health spending and universal health coverage in 188 countries, 2016-40. Lancet 2018; 391: 1783-1798.

3 Guenette JA, Webb KA, O'Donnell DE. Does dynamic hyperinflation contribute to dyspnoea during exercise in patients with COPD? Eur Respir J 2012; 40: 322-329.

4 Laveneziana P, Webb KA, Ora J, et al. Evolution of dyspnea during exercise in chronic obstructive pulmonary disease: impact of critical volume constraints. Am J Respir Crit Care Med 2011; 184: 1367-1373.

5 O'Donnell DE, Elbehairy AF, Faisal A, et al. Exertional dyspnoea in COPD: the clinical utility of cardiopulmonary exercise testing. Eur Respir Rev 2016; 25: 333-347.

6 Macklem PT. Therapeutic implications of the pathophysiology of COPD. Eur Respir J 2010; 35: 676-680.

7 Ambrosino N. Inspiratory muscle training in stable COPD patients: enough is enough? Eur Respir J 2018; 51: 1702285.

8 Charususin N, Gosselink R, Decramer M, et al. Randomised controlled trial of adjunctive inspiratory muscle training for patients with COPD. Thorax 2018; 73: 942-950.

9 Gosselink R, De Vos J, van den Heuvel SP, et al. Impact of inspiratory muscle training in patients with COPD: what is the evidence? Eur Respir J 2011; 37: 416-425.

10 Schultz K, Jelusic D, Wittmann M, et al. Inspiratory muscle training does not improve clinical outcomes in 3-week COPD rehabilitation: results from a randomised controlled trial. Eur Respir J 2018; 51: 1702000.

11 Laciuga H, Rosenbek JC, Davenport PW, et al. Functional outcomes associated with expiratory muscle strength training: narrative review. J Rehabil Res Dev 2014; 51: 535-546.

12 Weiner $\mathrm{P}$, Magadle R, Beckerman $\mathrm{M}$, et al. Comparison of specific expiratory, inspiratory, and combined muscle training programs in COPD. Chest 2003; 124: 1357-1364.

13 Miki K, Tsujino K, Edahiro R, et al. Exercise tolerance and balance of inspiratory-to-expiratory muscle strength in relation to breathing timing in patients with chronic obstructive pulmonary disease. J Breath Res 2018; 12: 036008.

14 Mayer AF, Karloh M, Dos Santos K, et al. Effects of acute use of pursed-lips breathing during exercise in patients with COPD: a systematic review and meta-analysis. Physiotherapy 2018; 104: 9-17.

15 Kojima M, Hosoda H, Date Y, et al. Ghrelin is a growth-hormone-releasing acylated peptide from stomach. Nature 1999; 402: 656-660.

16 Miki K, Kitada S, Miki M, et al. A phase II, open-label clinical trial on the combination therapy with medium-chain triglycerides and ghrelin in patients with chronic obstructive pulmonary disease. J Physiol Sci 2019; 69: 969-979.

17 American Thoracic Society. Standardization of spirometry, 1994 update. American Thoracic Society. Am J Respir Crit Care Med 1995; 152: 1107-1136.

18 Puente-Maestu L, Palange P, Casaburi R, et al. Use of exercise testing in the evaluation of interventional efficacy: an official ERS statement. Eur Respir J 2016; 47: 429-460.

19 Borg GA. Psychophysical bases of perceived exertion. Med Sci Sports Exerc 1982; 14: 377-381.

20 Laveneziana P, Albuquerque A, Aliverti A, et al. ERS statement on respiratory muscle testing at rest and during exercise. Eur Respir J 2019; 53: 1801214.

21 Hajiro T, Nishimura K, Tsukino $\mathrm{M}$, et al. Comparison of discriminative properties among disease-specific questionnaires for measuring health-related quality of life in patients with chronic obstructive pulmonary disease. Am J Respir Crit Care Med 1998; 157: 785-790.

22 Jones PW. St. George's Respiratory Questionnaire: MCID. COPD 2005; 2: 75-79.

23 Benjamini Y, Braun H. John W. Tukey's contributions to multiple comparisons. Ann Statist 2002; 30: 1576-1594. 
24 Neder JA, Arbex FF, Alencar MC, et al. Exercise ventilatory inefficiency in mild to end-stage COPD. Eur Respir J 2015; 45: 377-387.

25 Mota S, Guell R, Barreiro E, et al. Clinical outcomes of expiratory muscle training in severe COPD patients. Respir Med 2007; 101: 516-524.

26 Baz M, Haji GS, Menzies-Gow A, et al. Dynamic laryngeal narrowing during exercise: a mechanism for generating intrinsic PEEP in COPD? Thorax 2015; 70: 251-257.

27 Brancatisano T, Collett PW, Engel LA. Respiratory movements of the vocal cords. J Appl Physiol Respir Environ Exerc Physiol 1983; 54: 1269-1276. 